\title{
Event extraction on PubMed scale
}

\author{
Filip Ginter ${ }^{1 *}$, Jari Björne ${ }^{1,2}$, Sampo Pyysalo ${ }^{3}$ \\ From Workshop on Advances in Bio Text Mining \\ Ghent, Belgium. 10-11 May 2010
}

There has been a growing interest in typed, recursively nested events as the target for information extraction in the biomedical domain. The BioNLP'09 Shared Task on Event Extraction [1] provided a standard definition of events and established the current state-of-the-art in event extraction through competitive evaluation on a standard dataset derived from the GENIA event corpus.

We have previously established the scalability of event extraction to large corpora [2] and here we present a follow-up study in which event extraction is performed from the titles and abstracts of all $17.8 \mathrm{M}$ citations in the 2009 release of PubMed. The extraction pipeline is composed of state-of-the-art methods: the BANNER named entity recognizer [3], the McClosky-Charniak domainadapted parser [4], and the Turku Event Extraction System [5], the winning entry of the Shared Task.

The resulting dataset consists of over 19.2M instances of $4.5 \mathrm{M}$ unique events, of which $2.1 \mathrm{M}$ instances of $1.6 \mathrm{M}$ unique events recursively involve at least two different named entities. This dataset is several orders of magnitude larger than any previous event extraction effort and - having been obtained by a demonstrably state-ofthe-art pipeline - represents the most accurate event extraction output achievable with presently available tools. Compiling the dataset was a technically challenging undertaking and required roughly 8,300 CPU-hours.

As the primary contribution of the study, we make the entire set of extracted events freely available at http:// bionlp.utu.fi, together with the output of the individual stages of the pipeline, such as $36.5 \mathrm{M}$ named entity instances and syntactic analyzes for all $20 \mathrm{M}$ sentences containing at least one named entity. This resource will facilitate future research related to biological event networks by providing a standard, publicly available, largescale dataset, avoiding the unnecessary duplication of efforts in executing the complex event extraction pipeline.

\footnotetext{
* Correspondence: ginter@cs.utu.fi

'Department of Information Technology, University of Turku, Finland
}

Full list of author information is available at the end of the article

\section{Author details}

${ }^{1}$ Department of Information Technology, University of Turku, Finland. ${ }^{2}$ Turku Centre for Computer Science (TUCS), Finland. ${ }^{3}$ Department of Computer Science, University of Tokyo, Japan.

\section{Published: 6 October 2010}

\section{References}

1. Kim JD, Ohta T, Pyysalo S, Kano Y, Tsujii J: Overview of BioNLP'09 Shared Task on Event Extraction. In Proceedings of the BioNLP 2009 Workshop Companion Volume for Shared Task ACL 2009, 1-9.

2. Björne J, Ginter F, Pyysalo S, Tsujii J, Salakoski T: Complex Event Extraction at PubMed Scale. Proceedings of ISMB'10 2010, 26(12):i382-i390.

3. Leaman R, Gonzalez G: BANNER: an executable survey of advances in biomedical named entity recognition. Proceedings of Pacific Symposium on Biocomputing 2008, 652-663.

4. McClosky D: Any Domain Parsing: Automatic Domain Adaptation for Natural Language Parsing. PhD thesis Department of Computer Science, Brown University 2009.

5. Björne J, Heimonen J, Ginter F, Airola A, Pahikkala T, Salakoski T: Extracting Contextualized Complex Biological Events with Rich Graph-Based Feature Sets. Computational Intelligence 2010.

doi:10.1186/1471-2105-11-S5-O2

Cite this article as: Ginter et al:: Event extraction on PubMed scale. BMC Bioinformatics 2010 11(Suppl 5):O2.

\section{Submit your next manuscript to BioMed Central and take full advantage of: \\ - Convenient online submission \\ - Thorough peer review \\ - No space constraints or color figure charges \\ - Immediate publication on acceptance \\ - Inclusion in PubMed, CAS, Scopus and Google Scholar \\ - Research which is freely available for redistribution \\ Submit your manuscript at www.biomedcentral.com/submit}

